\title{
De Novo Complex Translocation 9; 22; 19 (Variant Philadelphia+) in Myelodysplastic Syndrome with Excess Blasts: A Case Report
}

Zeeshan Ansar Ahmed*, Muhammad Shariq Shaikh, Areeba Khalid, Bushra Moiz and Tariq Moatter

Department of Pathology and Laboratory Medicine, Aga Khan University Hospital, Karachi, Pakistan

\begin{abstract}
We report adult patients with myelodysplastic syndrome with excess blast (MDS) by the world health organization (WHO) 2016 revision for classification of myeloid neoplasm group. The case had clinical and hematological peculiarities, which had rarely reported and described yet. The cytogenetic alterations were complex translocation 9:22;19 (Variant Philadelphia+) of Philadelphia chromosomes which appeared at different moments of the disease. The patient showed the $\mathrm{Ph}$ chromosome with disease transformation at diagnosis and unfortunately, further workup could not be performed as the patient succumbed to her illness and expired. The complex characterization with multiple translocations and the presence of Ph could be a marker of this form of MDS. The association of clinical and hematological findings suggests the possibility of a new group of adult MDS.
\end{abstract}

Keywords: Myelodysplastic syndrome; Philadelphia chromosome; Cytogenetic; Complex translocation

\section{Background}

Myelodysplastic syndromes (MDS) are cytogenetically heterogeneous disorders that can transform to acute myeloid leukemia. The genetic profile of this syndrome is not well studied; however there is a link between genetic aberrations and defects in gene expression. An infrequent chromosome anomaly is the presence of the Philadelphia $(\mathrm{Ph})$ chromosome in MDS. Clonal cytogenetic abnormalities are found in $30 \%-50 \%$ of primary MDS cases. The major types include monosomy 7, loss of the $Y$ chromosome, $\operatorname{del}(5 q)$, del(7q), del(11q), del (20q), and trisomy 8 [1]. There is a higher frequency of chromosomal aberrations in progressive stages of the disease, which indicates disease progression to a transformed stage. Various genetic aberrations occur during the clonal evolution of MDS; however, there are few reports of the appearance of the Philadelphia $(\mathrm{Ph})$ chromosome [1]. The incidence rate of $\mathrm{Ph}$ chromosome has been reported to be approximately $20 \%$ of CML cases (adult cases), and 5\% pediatric cases demonstrating acute lymphoblastic leukemia (ALL), and in $3 \%$ acute myeloblastic leukemia (AML) cases [2]. In MDS however, the Ph chromosome is rarely involved. In this case report we report a patient with $\mathrm{Ph}+$ MDS in which the simultaneous emergence of $\mathrm{t}(9 ; 22 ; 19)$ of the $\mathrm{Ph}$ chromosome coincided with complex translocation to long arms (q) of 19 chromosomes.

\section{Case Description}

A 47-year-old female was evaluated for 2 weeks history of generalized weakness, weight loss and bleeding from mouth. She had no significant past medical and surgical history except for packed red cell transfusion. Physical examination revealed pallor with no hepatosplenomegaly or lymphadenopathy. Laboratory findings were as follows: Hemoglobin (Hb) $9 \mathrm{~g} / \mathrm{dL}$, mean cell volume (MCV) 77.1 fL, mean cell hemoglobin (MCH) $22.7 \mathrm{pg}$, white blood cells (WBCs) $47 \times 10^{9} / \mathrm{L}$, and absolute neutrophils count (ANC) $21.6 \times 10^{9} / \mathrm{L}$, Platelets $218 \times 10^{9} / \mathrm{L}$ and corrected reticulocyte count $4.1 \%$. There was no evidence of basophilia or monocytosis. Peripheral blood smear showed hypochromic microcytic anemia with anisocytosis, nucleated red blood cells, neutrophilia and 19\% blast cells (Figure 1). Based on clinical history and complete blood count findings, bone marrow procedure was carried out for evaluation of Mydelodysplastic/ Myeloproliferative Neoplasm (MDS/MPN). Bone marrow aspirate was a moderately cellular specimen exhibiting trilineage hematopoiesis with normoblastic erythropoiesis, and active myelopoiesis however with decreased myeloid to Erythroid ratio (1:1) and 17\% myeloblasts. Adequate megakaryocytes were noted. Bone trephine biopsy revealed increased cellularity for age of around $80-85 \%$ and grade MF-2 reticulin fibrosis. Immunohistochemistry highlighted increased CD34 and Myeloperoxidase (MPO) positive blast cells (Figure 1). All these findings were consistent with myelodysplastic syndrome (excess blastII). Conventional cytogenetic workup using Giemsa-Trypsin banding showed following karyotype: 46, XY, t $(9: 22 ; 19)$ (q34; q11.2; q13.3) (20) (Figure 2). Estimated revised-International prognostic scoring system

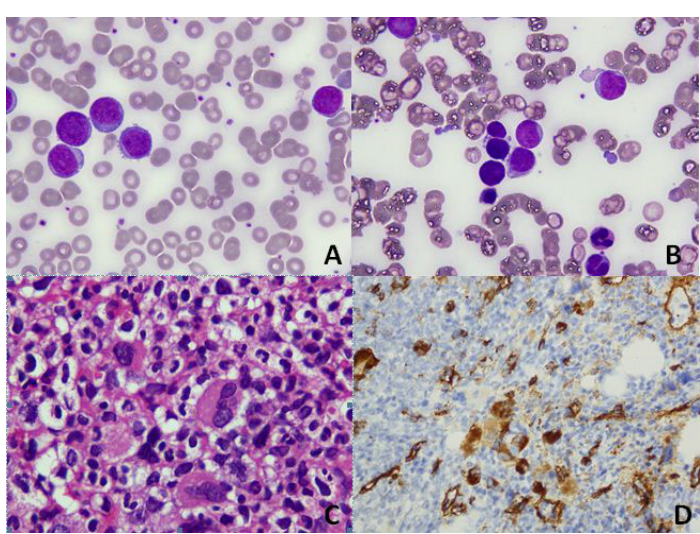

Figure 1: A: Peripheral blood film (at 100x), B: Bone marrow aspirate (at 100x), C: Bone trephine (H\&E section at 100x), D: CD34 stain at 40x.

*Corresponding author: Zeeshan Ansar Ahmed, FCPS (Haematology) Fellowship in Molecular Pathology, Department of Pathology and Laboratory Medicine, Aga Khan University Hospitals, Karachi Stadium Road, P.O.Box 3500 Karachi 74800, Pakistan, Tel: +922134864515; E-mail: Zeeshan.ansar@aku.edu

Received February 21, 2018; Accepted March 20, 2018; Published March 28 2018

Citation: Ahmed ZA, Shaikh MS, Khalid A, Moiz B, Moatter T (2018) De Novo Complex Translocation 9; 22; 19 (Variant Philadelphia+) in Myelodysplastic Syndrome with Excess Blasts: A Case Report. J Blood Lymph 8: 196. doi:10.4172/2165-7831.1000196

Copyright: (c) 2018 Ahmed ZA, et al. This is an open-access article distributed under the terms of the Creative Commons Attribution License, which permits unrestricted use, distribution, and reproduction in any medium, provided the original author and source are credited. 


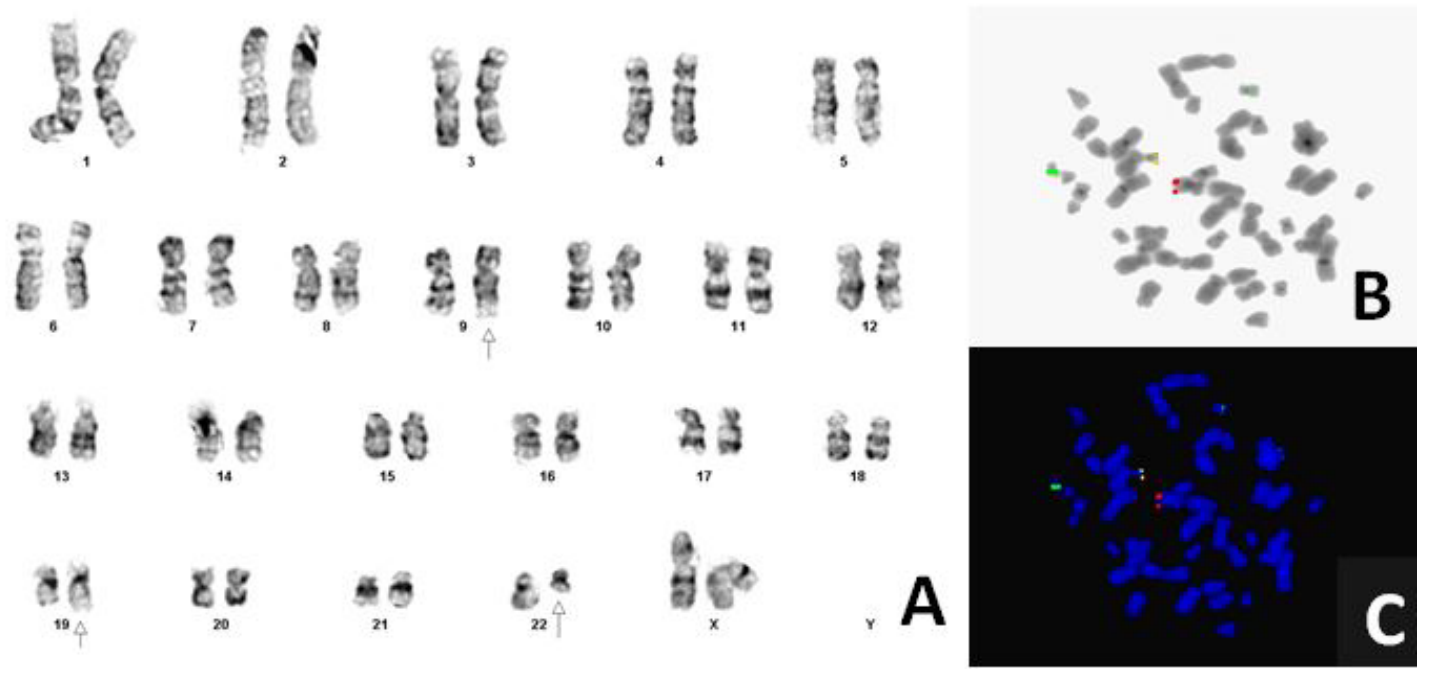

Figure 2: Bone marrow chromosome karyotype: 46, XY, t (9:22; 19) (q34; q11.2; q13.3) (20) and FISH revealed BCR/ABL1 fusion in 97\% of nuclei. Complex balanced translocation 9, 22 and 19 shows three break rearrangements (highlighted by the arrow heads). The segment on chromosomes 9 distal to $9 \mathrm{q} 34$ has been translocated onto chromosomes 22 at band 22q11.2; the segment on chromosome 22 distal to $22 q 11.2$ has been translocated onto chromosome 19 at $19 q 13.3$, and the segment of 19 distal to $19 q 13.3$ has been tranlocated onto chromosome 9 at $9 q 34$.

(R-IPSS) score was 3. (Hemoglobin $[9 \mathrm{~g} / \mathrm{dL}]=1$; platelets [218000/ $\mu \mathrm{L}]=0 ;$ absolute neutrophil count $[21600 / \mu \mathrm{L}]=0 ;$ cytogenetic $=1$; blast $=[19 \%]=2)$. Trilineage hematopoiesis, with excess blasts but interestingly, without basophilia, karyotypic, and molecular clonal evidence for $\mathrm{Ph}+$ disease. Unfortunately, further workup could not be performed as the patient succumbed to her illness and expired.

\section{Discussion}

Myelodysplastic syndrome is cytogenetically heterogeneous presenting with karyotypic abnormalities in about $50 \%$ of the patients. Gains and somatic loss of tumor suppressor result in impaired differentiation $[3,4]$. Translocation $(9 ; 22)$ is frequently observed in chronic myeloid leukemia (CML). In a WHO report (2016) classification of myeloid neoplasm emphasizes that CML BCR-ABL1+ phenotype must include significant myeloproliferation. The translocation $(9 ; 22)$ is rarely associated with dysplastic hematopoiesis,suggesting that it may have antagonistic disease-initiating role. In this case case report, we have described a $\mathrm{Ph}+\mathrm{MDS}$ patient.

Keung et al reported three de novo $\mathrm{Ph}+\mathrm{MDS}$ cases and reviewed 18 additional patients [5]. In contrast this case study was related to $\mathrm{Ph}+$ treatment-related disease and CMML case. About $48 \%$ of patients lack $\mathrm{Ph}+$ chromosomic abnormality and acquire translocation sequentially at disease progression. It is reported that $50 \%$ of patients present with $t$ $(9 ; 22)$ as the only abnormality. This disease exhibits high-risk features characterized by complex karyotype and elevated proportion of blast and thrombocytopenia. Other studies have also demonstrated that thrombocytopenia adversely impacts MDS outcome [6] Neukirchen et al. specifically. It is possible that $\mathrm{Ph}+\mathrm{MDS}$ exhibits similar prognostic variables as those observed in $\mathrm{Ph}$ negative MDS. A short median time to AML transformation of 7 months suggests that $\mathrm{Ph}+\mathrm{MDS}$ is a highly aggressive disease, especially if it is associated with complex karyotype and thrombocytopenia. In Keung et al. study [5], median time to AML progression was reported as 13 months. Ph chromosome is infrequently seen during the course of MDS. Late emergence of the Ph chromosome during the progress of MDS has been described in some case reports and reviews [7-9] and is referred to as substantiation supporting multistep leukemogenesis. The early event in leukemogenesis is ascribed to the clonal proliferation of stem cells, and the late acquirement of the $\mathrm{Ph}$ chromosome arises in neoplastic cells [10]. The element that few cases of $\mathrm{Ph}+\mathrm{MDS}$ have been reported to date is not conclusive evidence of low occurrence of this fusion in MDS patients.

FISH testing has accomplished to detect BCRABL fusion in MDS patients, lack of finding in normal karyotype study does not indicate definitive absence of this fusion [11,12]. Evaluation of reported cases shows that MDS patients concealing this chromosomal abnormality typically do not respond well to conventional treatments but do show a good response to imatinib therapy [13] Since imatinib is not consistently used in treatment of MDS patients, lack of $\mathrm{Ph}$ recognition in these patients may lead to improper treatment and thus put the patient's life at risk. Over-all, even if the findings of the studies specify the significance of $\mathrm{Ph}$ detection in MDS patients, they are not appropriate to illuminate the particular role of $\mathrm{Ph}$ in MDS patients. Hence, precise assessment of this chromosomal anomaly in MDS patients is suggested in future studies. Future directions should assess the role and probable appliance of action of TKIs in $\mathrm{Ph}+\mathrm{MDS}$ patients offering with low risk features.

\section{References}

1. Fenaux P, Morel P, Lai LJ (1996) Cytogenetic of myelodysplastic syndromes Semin Hematol 33: 127-138.

2. Raskind WH, Tirumali N, Jacobson R, Singer J, Fialkow PJ (1984) Evidence for a multistep pathogenesis of a myelodysplastisyndrome. Blood 63: 1318-1323.

3. Garcia-Manero G (2011) Myelodysplastic syndromes: 2011 update on diagnosis, risk-stratification, and management. American Journal of Hematology 86: 490-498.

4. Vardiman JW, Thiele J, Arber DA, Brunning RD, Borowitz MJ, et al. (2009) The 2008 revision of the World Health Organization (WHO) classification of myeloid neoplasms and acute leukemia: rationale and important changes. Blood 114 937-951.

5. Keung YK, Beaty M, Powell BL, Molnar I, Buss D, et al. (2004) Philadelphia chromosome positive myelodysplastic syndrome and acute myeloid leukemiaretrospective study and review of literature. Leukemia Research 28: 579-586.

6. Kantarjian H, Giles F, List A, Lyons R, Sekeres MA, et al. (2007) The incidence 
Citation: Ahmed ZA, Shaikh MS, Khalid A, Moiz B, Moatter T (2018) De Novo Complex Translocation 9; 22; 19 (Variant Philadelphia+) in Myelodysplastic Syndrome with Excess Blasts: A Case Report. J Blood Lymph 8: 196. doi:10.4172/2165-7831.1000196

Page 3 of 3

and impact of thrombocytopenia in myelodysplastic syndromes. Cancer 109: 1705-1714.

7. Neukirchen J, Blum S, Kuendgen A, Strupp C, Aivado M, et al. (2009) Platelet counts and haemorrhagic diathesis in patients with myelodysplastic syndromes. European Journal of Haematology 83: 477-482.

8. Tien HF, Wang CH, Chuang SM, Lee FY, Liu MC, et al. (1992) Characterization of Philadelphia-chromosome-positive acute leukemia by clinical, immunocytochemical, and gene analysis. Leukemia 6: 907-914.

9. Whang-Peng J, Henderson ES, Knutsen T, Freireich EJ, Gart JJ (1970) Cytogenetic studies in acute myelocytic leukemia with special emphasis on the occurrence of Ph1 chromosome. Blood 36: 448-457.

10. Fialkow PJ, Martin PJ, Najfeld V, Penfold GK, Jacobson RJ, et al. (1981)
Evidence for a multistep pathogenesis of chronic myelogenous leukemia. Blood 58: 158-163.

11. Vardiman JW, Thiele J, Arber DA, Brunning RD, Borowitz MJ, et al. (2009) The 2008 revision of the World Health Organization (WHO) classification of myeloid neoplasms and acute leukemia: rationale and important changes. Blood 114: 937-951.

12. Drummond MW, Lush CJ, Vickers MA, Reid FM, Kaeda J, et al. (2003) Imatinib mesylate-induced molecular remission of Philadelphia chromosome-positive myelodysplastic syndrome. Leukemia 17: 463-465.

13. Nybakken GE, Bagg A (2014) The genetic basis and expanding role of molecular analysis in the diagnosis, prognosis, and therapeutic design for myelodysplastic syndromes. J Mol Diagn 16: 145-158. 